\title{
LA CLASIFICACIÓN INTERNACIONAL PARA LA PRÁCTICA DE ENFERMERÍA COMO EJEMPLO DE INVESTIGACIÓN EN RED
}

\author{
INTERNACIONAL CLASIFICATION FOR NURSING PRACTICE US \\ AN EXAM PLE OF NETWORK RESEARCH
VERÓNICA BEHN THEUNE*, XIMENA BASSO**, PATRICIA CID HENRÍQUEZ***, MÓNICA CRUZ PEDREROS****,JULIA RAMÍREZ CASTILLO*****, MATILDE TORRUELLA PUENTES******

\begin{abstract}
RESUMEN
Se presentan las fortalezas y debilidades del trabajo investigativo en red a la luz de la experiencia de los autores en la investigación detérminos para la Clasificación Internacional de la Práctica de Enfermería (CIPE®).
\end{abstract}

Palabras claves: Investigación, red, CIPE $尺$.

\section{ABSTRACT}

The strengths and weaknesses of the research in network by the experience of the authors in research of terms for the International Classification for Nursing Practice (ICNP $®$ ) is presented.

Keywords: Network, research, ICNP ${ }^{\circledR}$.

Recepcionado: 28.04.2004 Aceptado: 04.10.2004

\section{INTRODUCCIÓN}

El trabajo investigativo en red ha sido reconocido en diferentes ámbitos como una forma de incrementar el valor de los resultados obtenidos en esta tarea. Es así como se establecen flujos de información y retroalimentación permanente que, a través del dar y recibir de los integrantes de la red, posibilitan intercambiar experiencias y potenciar la generación del conocimiento, afirmando lazos en torno a problemas comunes, sin perder la autonomía. La red permite crear una atmósferadeapoyo, cooperación y colaboración con el reconocimiento del potencial del otro (Sena, 2003). Al aunar esfuerzos en un poder compartido, en un mundo globalizado, se evita la duplicidad dela información y con ello selogra mejorar el costo beneficio del trabajo.

La red es un vínculo, lazo, unión, articulación einteracción entrepersonas, gruposeinstituciones, quienes participan activamente constituyendo los actores primarios de la red (García, Herrero \& Rusitu, 1995; CONACE, 2001). Rovere (en Sena 2003) identifica cinco niveles que se articulan en el trabajo en red, como son: Reconocer, Conocer, Colaborar, Cooperar y Asociarse. Estosniveles funcionan sobre la base valores de la aceptación, el interés, la reciprocidad, la solidaridad y la confianza.

En la metodología del trabajo en red sereconocen algunas etapas quehacen posibleque la red avance, según señala Elkaim (1992):

\footnotetext{
* Enfermera, Docente Facultad de M edicina, Universidad de Concepción-Chile. E-mail: vbehn@udec.cl

** Enfermera CESFAM O'Higgins, Concepción-Chile.

*** Enfermera, Docente Facultad de M edicina, Universidad de Concepción-Chile. E-mail: patcid@udec.cl **** Enfermera, Docente Facultad de M edicina, Universidad de Concepción-Chile. E-mail: mcruz@udec.cl ***** Enfermera, Docente Facultad de M edicina, Univ. de Concepción-Chile. E-mail: jramirez@udec.cl ****** Enfermera, Docente Facultad de M edicina, Univ. de Concepción-Chile. E-mail: mtorruel@udec.cl Dirección: Universidad de Concepción, Casilla 160-C, Concepción. Fax: 41-228353.
} 
- Orientación de las responsabilidades de los diferentes actores.

- Difusión y sensibilización hacia el medio en que se desarrolla la investigación con el fin de lograr los apoyos que facilitan el intercambio y progreso sostenido hacia el logro de las metas propuestas.

- Compromiso formal delosparticipantespara contribuir activamente en el flujo de la red.

- Mantención del diálogo real y virtual, sin interrumpir las conexiones entre los participantes.

- Monitoreo del avance, para mantener el compromiso y demostrar los logros.

\section{LA CIPE ${ }^{\circ}$ COMO INVESTIGACIÓN EN RED}

La tarea de desarrollar una Clasificación Internacional para la Práctica de Enfermería (CIPE ${ }^{\circledR}$ ) es la respuesta a la necesidad reconocida hace más de una década por el Consejo Internacional de Enfermeras (CIE), con la finalidad de crear un vocabulario de términos estructurado para describir y organizar la práctica deenfermería (Clark, 1996; Ruland, 2001). La posibilidad de nombrar lo que las enfermeras/os hacen, los diagnósticos de enfermería de nuestros clientes y las interven- ciones deenfermería, constituyeun valor fundamental quepermitequela enfermeríacomo disciplina se haga visibley siga en desarrollo. La base de datos que se crea con este instrumento apoyará las decisiones en salud, las investigaciones en enfermería y la docencia (Warren \& Coenen, 1998). Además, permitirá unificar cuidados, compararlos y apoyar la evidencia del resultado. Una constanteactualización al Programa CIPE $®$ del Consejo Internacional de Enfermeras se ofrece en la página web del CIE: www.icn.ch

Con el trabajo de crear un lenguaje común de enfermería, comienza a vivirse una experienciaquedebía unir y vincular, en lo posible, a todas las enfermeras/os del mundo para ge nerar resultados válidos. Se hizo evidente que una red de investigación podría aportar a la CIPE $®$ resultados exitosos. Su primer producto sematerializó en un documento, CIPE $®$ alfa, elaborado en red, cuyo contenido es la identificación de fenómenos de enfermería, acciones de enfermería y resultados, clasificados en una estructura propia. Actual menteestá en circulación su versión aumentaday corregida, que corresponde a CIPE $囚$, beta 2 (CIE 2001).

Los diferentes actores interesados en participar en la investigación debieron unirsea una estructura con enlaces claros, a manera de red, tal como se observa en el diagrama siguiente:

Diagrama 1. Estructura de la red de intercomunicación en CIPE ${ }^{\circledR}$

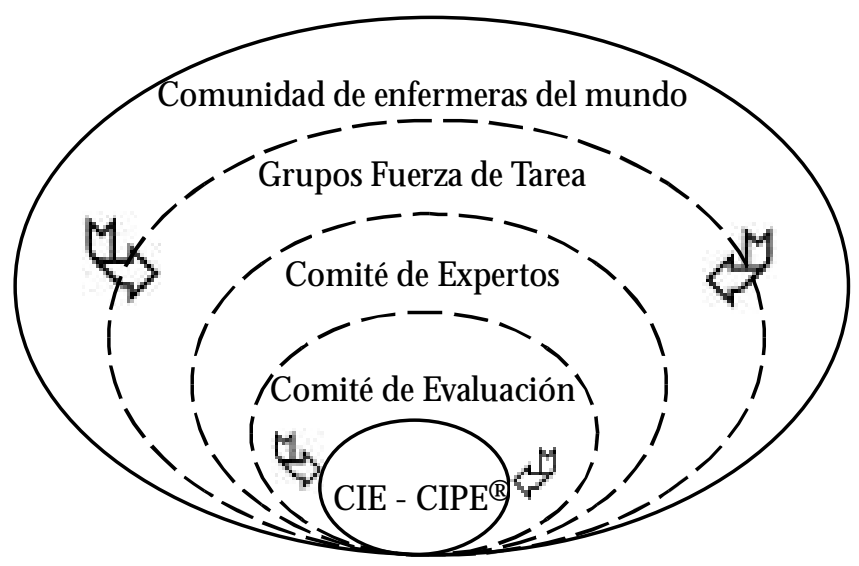

Fuente: Artículo “La clasificación internacional para la práctica en enfermería como ejemplo de Investigación en red”. 
El grupo más numeroso de la red lo constituyen las/los colegas que integran la comunidad de enfermeras/os delos países afiliados al $\mathrm{CIE}$, y aquellos que deseen contribuir con su experiencia a esta tarea.

Luego están los Grupos Fuerza deTarea de cada país participante, quienes organizan, observan y analizan las actividades de enfermería, para adoptar y difundir las metodologías que permitirán develar el fenómeno del dominio de la profesión queemana dela práctica.

Un grupo selecto de enfermeras/os representantes de los diferentes países participantes constituye un Comitéde Expertos, quetienela tarea de analizar el trabajo realizado por el Grupo Fuerza de tarea y expresar su acuerdo o desacuerdo en aspectos de pertinencia para que los términos identificados puedan ser incorporados a la CIPE $®$.

Finalmente existe el grupo de enfermeras que conforman el Comité Internacional de Evaluación, compuesto por 6 integrantes de diferentes continentes, quienes, de conjunto con el staff del CIE, deben finalmente sancionar las propuestas que se incluyen en el do-

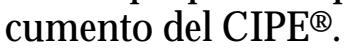

¿Cómo funciona la red?

- La comunidad de enfermeras abastece al Grupo Fuerza de Tarea de situaciones derivadas de su actividad práctica para iniciar la investigación focalizada en estosfenómenos de enfermería.

- El Grupo Fuerza de Tarea procesa la información, la tamiza, la depura eidentifica una primera propuesta de término de fenómeno con sus características esenciales. Este material elaborado vuelve a la comunidad de enfermeras para la validación inicial, en que participa un amplio número de colegas familiarizados con el fenómeno a validar.

- La retroalimentación que recibe el Grupo Fuerza de Tarea permite afinar el producto y validar por segunda vez las características del término. En esta oportunidad el Grupo Fuerza de Tarea realiza la validación con el aporte de la literatura y de la búsqueda de información en bibliotecas virtuales.

- El producto depurado y aceptado a este nivel es enviado al staff central del programa CIPE ${ }^{\circledR}$ acompañado de la estadística y las referencias quehan permitido su validación final.

- El staff central del Programa CIPE ${ }^{\circledR}$ del Consejo Internacional de Enfermeras lo distribuye a expertos registrados con anterioridad, a través de una convocatoria internacional.

- Las respuestas de los Expertos ofrecen un informe detallado, querecomienda o rechaza la incorporación del término a la Clasificación Internacional. Estas respuestas son analizadas por el Comité Internacional de Evaluación, quien final menteacepta, rechaza o solicita la revisión del término a quienes lo elaboraron 0 a quienes estimen indicados.

- El término aceptado es incorporado por el

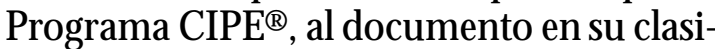
ficación correcta. El término rechazado vuelve al Grupo Fuerza de Tarea para su eventual reformulación o para reorientar el trabajo.

\section{COMENTARIOSY SUGERENCIAS}

La red CIPE $® s e h a$ formado y hatenido éxito gracias a que el grupo de profesionales participantes tiene una vinculación natural y ha desarrollado un sentido de pertenencia, el cual es fomentado por las agrupaciones gremiales de los respectivos países. La necesidad sentida del lenguaje propio, junto a la convicción del valor individual del aporte de las enfermeras a la tarea propuesta, refuerza su compromiso. La flexibilidad de los vínculos formados entre la comunidad de enfermeras y los Grupo Fuerza de Tarea ha fomentado la inclusión de nuevas colegas, que aportan conocimientos específicos de su ámbito de trabajo en un ambiente de contacto periódico y real. Para las validaciones delo elaborado tam- 
bién se ha mantenido la flexibilidad de incorporar a esta etapa del trabajo a un número cada vez mayor de profesionales de enfermería, a través de canales de comunicación de variada índole y eficacia, según tecnología disponible en los respectivos lugares.

Elkaim (1992), al respecto, afirma que los aportes de una red sólo son posibles si la red cumple con las siguientes condiciones básicas:

- Espacio de igualdad.

- Participación voluntaria.

- Relaciones horizontales, sin jerarquía.

- Facilitador democrático.

- Reconocimiento de saberes y aportes.

- Flexibilidad en la comunicación, en el ajuste de objetivos, en la incorporación de nue vos miembros a la red.

- A mayor diversidad de la red mayor creatividad.

- Aceptación del cambio cultural en la gestión: no es un fin en sí, sino una forma de trabajo.

- Contacto periódico entre los miembros.

Por otro lado, también es necesario reconocer que existen factores obstaculizadores del trabajo en red, que Cubillos (1999) identifica como los siguientes:

- Objetivos poco claros.

- M etodologías poco claras.

- Apego a la jerarquía.

- Falta de perseverancia.

- Expectativas de éxito inmediatos.

- Herramienta de comunicación inoperante (déficit detecnología y falta decapacitación).

En nuestra experiencia, la investigación en red ha generado un número importante de nuevos términos que tienen relación con el trabajo de la enfermera con familias (Alvarez, Figueroa, M uñoz \& M oya, 2000; Behn, Cid, Ramirez, Torruella, 2000; Colegio de Enfermeras de Chile A.G., 1996). Algunos de los términos elaborados a través de la investigación en red son: Crisis familiar, Disfunción familiar, Desarrollo psicomotor, Violencia in- trafamiliar, Duelo familiar, Adaptación familiar, Desintegración familiar. Las enfermeras participantes en nuestra red fueron motivadas para profundizar y difundir los conocimientos adquiridos durante el trabajo investigativo. Es así como se originaron cursos y talleres deactualización en temáticas estudiadas y analizadas en el proceso de elaboración de los términos para el trabajo con familias. Estas actividades de educación continua interesaron de preferencia a las enfermeras que deseaban mejorar la atención de enfermería en el nivel primario de atención y permitieron que percibieran con mayor fuerza su participación valiosa en la tarea.

Creemos que esta experiencia en red podría aplicarse también en grupos interdisciplinarios quetrabajan líneas deinvestigación comunes, así como al nivel de estudiantes de pregrado, para motivar el intercambio de los jóvenes talentos.

\section{AGRADECIMIENTOS}

El Grupo de Investigación CIPE dela Universidad de Concepción desea agradecer el apoyo y aporte valioso, constante, desinteresado y comprometido de las colegas ex docentes del Departamento de Enfermería de la Universidad de Concepción, Sra. Rina Aguayo, Srta. M aría Figueroa, Sra. Olga M artínez, Sra. Olga Polanco y Sra. Pilar Zapatero. Al igual deseamos agradecer la colaboración de las colegas que ayudaron a validar con su experiencia los términos trabajados. Finalmente agradecemos a la Sra. Ingrid Ramdohr, GerenteZona Sur deLaboratorio Saval, por acogernos en un espacio tranquilo y motivador para el estudio.

\section{REFERENCIAS BIBLIOGRÁFICAS}

Alvarez, M.; Figueroa, M.; Muñoz, L.A. \& Moya, C. (2000). Proyecto de Clasificación Internacional de la Práctica de Enfermería. Enfermería, 10: 24-29. Behn, V.; Cid, P.; Ramirez, J. \& Torruella M. (2000). 
Diagnósticos deEnfermería en Familias y propuesta deintervenciones. H orizonte deEnfermería, 10 (1): 24-30.

CIE (2001). Clasificación Internacional para la Práctica deEnfermería(CIPE). CIPE Versión Beta 2. Ginebra, Suiza.

Clark, J. (1996). How Nurses can participate in the development of an ICNP. International Nursing Review, 43 (6): 171-174.

Colegio de Enfermeras de ChileA.G. (1996). Proyecto Clasificación de la Práctica de Enfermería, aporte de Chile. Documento elaborado con apoyo de la Fundación W. K. Kellogg.

Cubillos, J. (1999). La universidad abierta en red. Comisión Económica paraAmérica Latina. Documento de CEPAL.

CONACE (2001). La Red Social. Modelo operativo. Sistema Comunal de Prevención de Drogas. Santiago de Chile.

Elkaim, M . (1992). Red de redes. Ed. Gedisa. España.
Garcia, E.; Herrero, J. \& Rusitu, G. (1995). El estudio del apoyo social: perspectivasteóricas. SerieBiblioteca de Ciencias Sociales. PPV. Barcelona, España.

Ruland, C.M . (2001). Evaluating the Beta version of the International Classification for N ursing Practice for domain completeness, applicability of its axial structure and utility in clinical practice: a Norwegian Project. International Nursing, 48 (1): 9-16.

Sena R.R. (2003). La Red de Enfermería en América Latina (REAL). En Wright, G., Chisman A., Gallegos E., Salazar B., Espinoza S., Alonso M . Los nuevos programas de Doctorado en Enfermería y su contribución en la reducción de la demanda de drogas en América Latina: Retos y perspectivas. Washington D.C.; U.S.; M onterey, M éxico. OEA.

Warren J.J \& Coenen, A. (1998). International Classification for Nursing Practice (ICNP): M ost frequently asked questions. Journal of theAmerican M edical Informatics Association, 5 (4): 335-336. 